\title{
INVESTIGATION OF LOCAL TRAPPING STATES IN ORGANIC MOLECULAR CRYSTALS BY METHOD OF THERMALLY MODULATED SPACE-CHARGE LIMITED CURRENT.
}

\author{
I. Muzikante and E.A. Silinsh
}

Institute of Physical Energetics, Latvian Ac. Sci., 21 Aizkraukles Str., 1006 Riga, Latvia

(Received March 29, 1995; in final form May 15, 1995)

Dedicated to Professors Krzysztof Pigoń, Józef W. Rohleder and Zdzistaw Ruziewicz on the occasion of their Y0th birthdays*

The method of thermally modulated space-charge limited current has been used for studying local charge carrier trapping state energy spectra in vacuum evaporated thin films and Langmuir-Blodgett multilayers of different organic molecular crystals. In case of evaporated thin polycrystalline layers of pentacene four sets of local trapping states in energy range from $E_{\mathrm{t}}=0.35$ to $0.06 \mathrm{eV}$ were observed. In evaporated thin layers of dimethylaminobenzylidene indandione-1,3 five sets of trapping states from $E_{\mathrm{t}}=0.59$ to $0.28 \mathrm{eV}$ were detected. In the evaporated layers of indandione-1,3 pyridinium betaine only a single set of very shallow trapping states close to the conductivity level was observed. In the Langmuir-Blodgett multilayers of vanadyl phthalocyanine and indandione-1,3 pyridinium betaine the activation energy $E_{\mathrm{a}}$ dependence on applied voltage $U, E_{\mathrm{a}}(U)$, for bulk conductivity is not step-like as in case of evaporated layers, but of rather complicated, oscillatory shape. Such character of the $E_{\mathrm{a}}(U)$ dependence may be explained by the specific quantum well structure of the Langmuir-Biodgett multilayers and spatially non-uniform trap distribution. However, for in-plane conductivity of indandione-1,3 pyridinium betaine films the $E_{\mathrm{a}}(U)$ dependence is step-like and two sets of trapping states at $E_{\mathrm{t}}=0.45 \pm 0.03 \mathrm{eV}$ and $E_{\mathrm{t}}=0.18 \pm 0.03 \mathrm{eV}$ were obtained. In case of bulk conductivity of the Langmuir-Blodgett layers the activation energy $E_{\mathrm{a}}$ at sufficiently high electric fields drops down to zero and the charge carrier transport switches to a "trap-free" regime.

PACS numbers: $72.20 . \mathrm{Jv}, 72.80 . \mathrm{Le}, 73.50 . \mathrm{Gr}, 73.61 . \mathrm{Ph}, 73.40 . \mathrm{Rw}$

*Submitted on invitation of the Institute of Physical and Theoretical Chemistry, Technical University of Wrocław, Wrocław, Poland. 


\section{Introduction}

It has been shown that different structural defects in organic molecular crystals (OMC) form local electronic states of polarization origin: trapping states (traps) and scattering states (antitraps) for charge carriers as well as excitons. The most feasible approximation of energy spectra for such local states is found to be the Gaussian distribution model [1-3].

Among the variety of experimental methods for studying the local states the most appropriate are the methods of space-charge limited current (SCLC) [1, 2]. As a result of approximation of experimental characteristics the main parameters of a given set of traps distribution such as total density traps $N_{\mathrm{t}}$, distribution parameter $\sigma$ as well as energy depth $E_{\mathrm{t}}$ may be determined. The conventional SCLC method is most appropriate for studying shallow trap distributions. Recently there has been developed the method of thermally modulated SCLC (TM-SCLC) which is based on careful measurements of the dark conductivity activation energy $E_{\mathrm{a}}$ dependence on voltage $U: E_{\mathrm{a}}=f(U)[4-7]$. The method yields the parameters $E_{\mathrm{t}}$, $N_{\mathrm{t}}$ and $\sigma$ and provides also higher resolution of energy spectra in comparison with the conventional SCLC method [8].

In these studies we have investigated the energy spectra and distribution parameters of local charge carrier trapping states for different classes of OMC. We have also applied the TM-SCLC method for studying samples of different structure (vacuum evaporated films and Langmuir-Blodgett (LB) multilayers).

\section{Objects of investigation}

The paper presents the experimental results of the TM-SCLC method for three different classes of organic molecular compounds.

\subsection{Pentacene}

Pentacene $(\mathrm{Pc})$ belongs to the class of polyacenes with lattice type $\mathrm{A}$ of aromatic crystals [1]. Pentacene has been for decades the most popular object for detailed studies of electronic and excitonic processes in OMC [1, 2, 9-11].

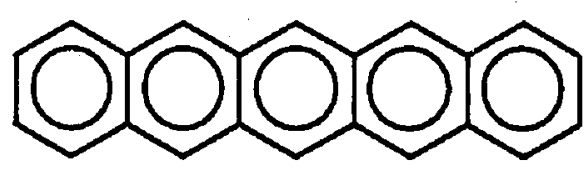




\title{
2.2. Phthalocyanine
}

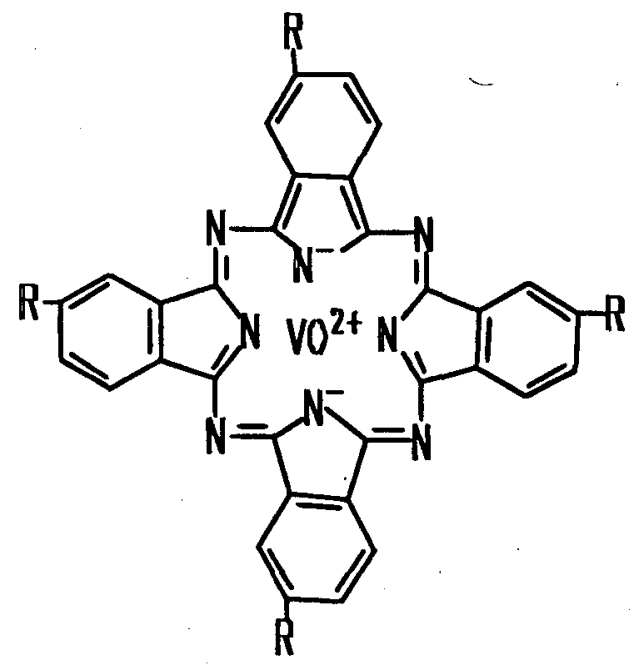

\begin{abstract}
Phthalocyanine (Phc), possessing a cyclic polyconjugated molecular structure, often serves as a model prototype of chemically similar, biologically important porphins. In our case an amphiphilic surface active compound tetra(octadecylaminosulphanyl) vanadyl phthalocyanine (VOPhc) with 4 aliphatic tails, where $\mathrm{R}=$ $\mathrm{SO}_{2} \mathrm{NHC}_{18} \mathrm{H}_{37}$, was investigated in LB films [12, 13].
\end{abstract}

2.3. Indandione-1, 3 pyridinium betaine (IPB) and dimethylaminobenzylidene indandione-1,3 (DMABI)

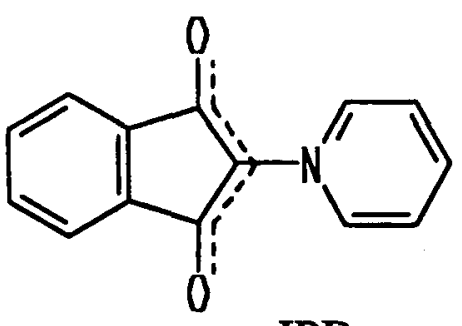

IPB

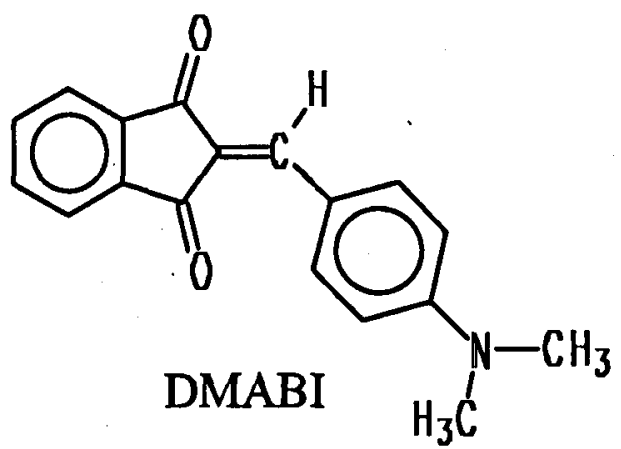

DMABI

They belong to the series of highly polar derivatives of the indandione-1,3. These compounds were investigated as vacuum evaporated polycrystalline films as well as LB films with aliphatic tail, containing 17 carbon atoms (C17IPB):

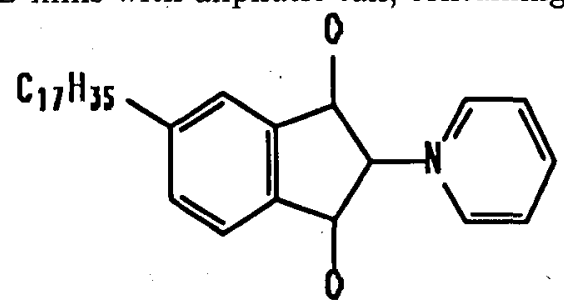

IPB and DMABI are excellent photoconductors $[1,3,14,15]$ and promising for non-linear optics $[14,16]$.

The samples of the given OMC were prepared as thin layers by vacuum evaporation as well as by the LB techniques. The samples were deposited on quartz, 
sapphire, glass or silicon substrates. For studying bulk conductivity the "sandwich" type samples $\mathrm{Me} / \mathrm{OMC} / \mathrm{Me}$ were prepared. $\mathrm{Au}, \mathrm{Cr}, \mathrm{Bi}$ and $\mathrm{Al}$ were used as electrodes for injection of holes in the OMC samples. It should be noticed that the charge carrier injection from electrodes in organic materials cannot be related with work functions of metal and organic crystals, but actually depends on the specific properties of the metal/organic interface and mechanisms of charge carrier injection [1].

The evaporation temperature of Pc films was $T=230^{\circ} \mathrm{C}$, of IPB films $T=$ $257^{\circ} \mathrm{C}$ and of DMABI films $T=150^{\circ} \mathrm{C}$. The thickness of vacuum evaporated polycrystalline films was of the order of $0.3-1 \mu \mathrm{m}$. The in-plane conductivity of LB films was measured for surface cell $\mathrm{Au} / \mathrm{OMC} / \mathrm{Au}$ with distance between electrodes 60-150 $\mu \mathrm{m}$.

LB multilayer assemblies of $Z$ - or $Y$-type were deposited with different number of monolayers $(N=4-50)$. The deposition method has been described in Refs. $[12,13,16]$.

\section{Experimental. Description of the TM-SCLC method}

The TM-SCLC method was developed by S. Nešpurek and co-authors [4-7] who introduced a new variant of SCLC approach which is often called thermally modulated SCLC spectroscopy of traps. This method provides a direct measurement of the energy spectra of trapping states and needs no initial input parameters like conventional SCLC approach. The method is based on the model of the Gaussian distribution of energy spectra $h(E)$ of local states $[1,3]$ :

$$
h(E)=\left[N_{\mathrm{t}} /(\sigma \sqrt{2 \pi})\right] \exp \left[-\left(E-E_{\mathrm{t}}\right)^{2} / 2 \sigma^{2}\right],
$$

where $E_{\mathrm{t}}$ is the energetic depth of a set of traps, $N_{\mathrm{t}}$ is the total density of traps in the set and $\sigma$ is the corresponding parameter of distribution of traps.

The TM-SCLC method applies the so-called "fractional cooling" technique [4]. At the given voltage $U=$ const the sample is slowly cooled $(\approx 0.05 \mathrm{~K} / \mathrm{s})$ and the corresponding current-temperature $I=f(T)$ dependence is measured in the SCLC regime under monopolar injection of holes from the positive electrode. The temperature range in the cooling cycle should not exceed $c a \cdot 10^{\circ} \mathrm{C}$. The activation energy $E_{\mathrm{a}}$ at the given voltage $U$ is determined as

$$
E_{\mathrm{a}}=k_{\mathrm{B}} \Delta \ln I / \Delta(1 / T),
$$

where $k_{\mathrm{B}}$ is the Boltzmann constant.

After the cooling cycle the temperature is slowly increased $(\approx 0.1 \mathrm{~K} / \mathrm{s})$ reaching several degrees above initial one (in order to depopulate the filled traps), and then the cycle is repeated once more at another value of $U$, and so on $[4,11,12]$. The cyclic repetition of the temperature $T$ and voltage $U$ is schematically shown in Fig. 1. As a result, one obtains the $E_{\mathrm{a}}(U)$ dependence in the form of step-like structure which directly reflects the energy spectrum of trapping states. The activation energy $E_{\mathrm{a}}$ in this case characterizes the position of the quasi-Fermi level $E_{\mathrm{F}}$ which, in turn, is determined by the level of filling the traps as a function of the injecting voltage $U$. It may be shown that at the voltage $U=(1 / 2) U_{\text {TFL }}$ (where $U_{\mathrm{TFL}}$ is the trap filled limit voltage) the value of $E_{\mathrm{a}}$ becomes equal to 


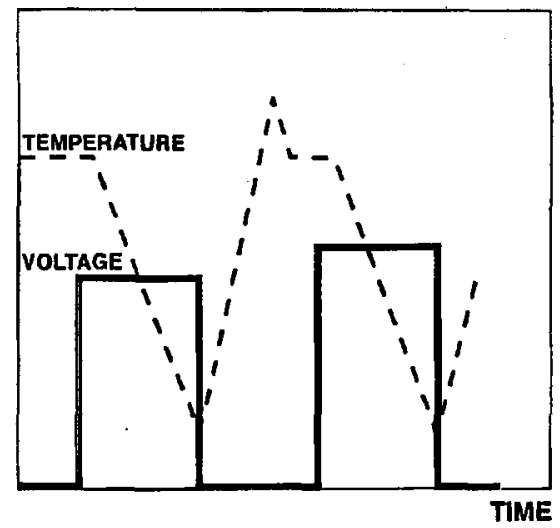

Fig. 1. Schematic diagram of the procedure of experimental measurements illustrating the TM-SCLC method.

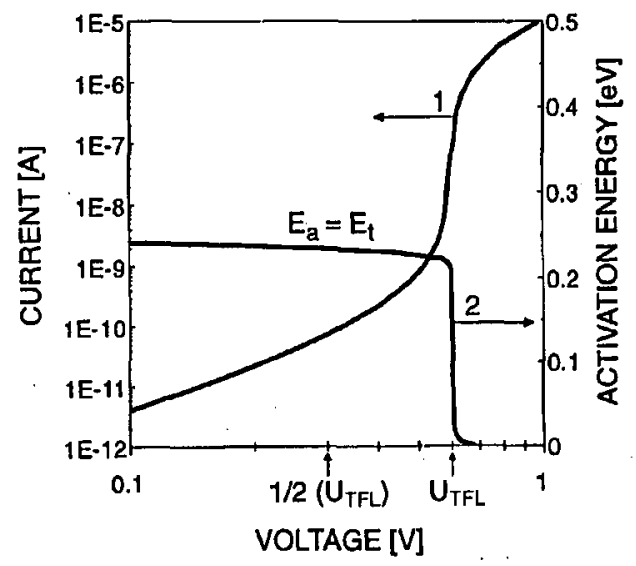

Fig. 2. The calculated current voltage (CV) characteristic (1) and corresponding dependence of activation energy on applied voltage (2) according to the TM-SCLC method with following parameters: $E_{\mathrm{t}}=0.23 \mathrm{eV}, N_{\mathrm{t}}=1 \times 10^{14} \mathrm{~cm}^{-3}, \sigma=0.015 \mathrm{eV}$, thickness of sample $L=1 \mu \mathrm{m}$ and temperature $T=200 \mathrm{~K}$.

$E_{\mathrm{t}}$, viz. $E_{\mathrm{a}}=E_{\mathrm{t}}$, and remains constant until all traps of the given set are filled $\left(U=U_{\mathrm{TFL}}\right)$, after which it jumps to zero value or to the next step of the $E_{\mathrm{a}}(U)$ dependence (see Fig. 2). Besides the $E_{t}(E)$ spectrum the method allows, under certain conditions, to evaluate from the $E_{\mathrm{a}}(U)$ dependence also the parameters $\sigma$ and $N_{\mathrm{t}}$.

Samples were situated in vacuum cryostat ( $p=1 \times 10^{-6}$ torr) and thermoregulator was used for thermal measurements from $100 \mathrm{~K}$ till $320 \mathrm{~K}$. The sample was provided by a digitally controlled voltage supply over a range up to $100 \mathrm{~V}$ and the current was measured by electrometer in range from $1 \times 10^{-13}$ to $1 \times 10^{-3} \mathrm{~A}$. 


\section{Local trapping states in polycrystalline samples}

In case of vacuum evaporated films the CV-characteristics in the SCLC regime were either non-linear (IPB, DMABI) or S-shaped (Pc) as one can expect in case of Gaussian distribution of local trapping states [1]. The dependencies of $E_{\mathrm{a}}(U)$ are step-like in case of Pc [11] and DMABI (see Fig. 3 and Fig. 4) and

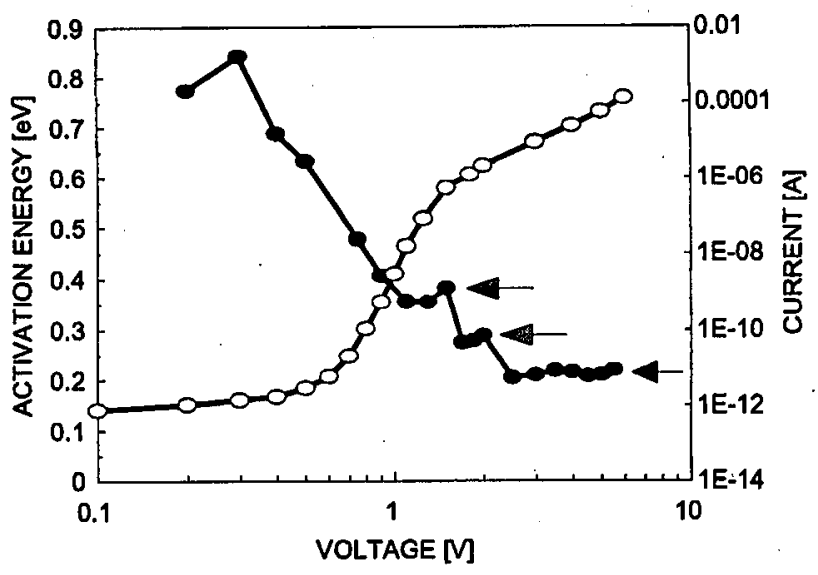

Fig. 3. Experimental CV-characteristic (o) and $E_{\mathrm{a}}(U)$ dependence $(\bullet)$ of the Pc vacuum evaporated thin films in a "sandwich" type cell $\mathrm{Au} / \mathrm{Pc} / \mathrm{Al}$. The temperature range $T=$ $300-290 \mathrm{~K}$; the thickness of the film $L=0.4 \mu \mathrm{m}$; injection of holes from positive $\mathrm{Au}$ electrode. The arrows show the energy depth $E_{\mathrm{t}}$ of three sets of trap distribution.

correspond the theory of the TM-SCLC method.One of the criteria of the SCLC regime is the inverse power law dependence of the current on the sample thickness. We have studied this dependence in detail for a number of evaporated layers, especially for IPB [1]. However, the SCLC theory of the Gaussian distribution of traps gives independent criteria. Thus, in case of the Gaussian distribution the CV-characteristics in the SCLC regime yield either S-shaped (in case of a single set of traps) or step-like CV-curves (in case of several sets of traps). Also the $E_{\text {a }}$ dependence in the TM-SCLC regime shows a step-like behaviour (see Fig. 2).

As may be seen from Fig. 3 and Fig. 4 several sets of traps emerge in these thin films. In case of $\mathrm{Pc}$ the four sets of traps with energies $E_{\mathrm{t}}^{1}=0.35 \pm 0.05 \mathrm{eV}$, $N_{\mathrm{t}}^{1}=3 \times 10^{14} \mathrm{~cm}^{-3}, E_{\mathrm{t}}^{2}=0.28 \pm 0.05 \mathrm{eV}, N_{\mathrm{t}}^{2}=4 \times 10^{14} \mathrm{~cm}^{-3}, E_{\mathrm{t}}^{3}=0.21 \pm 0.03 \mathrm{eV}$, $N_{\mathrm{t}}^{3}=1 \times 10^{15} \mathrm{~cm}^{-3}$ and $E_{\mathrm{t}}^{4}=0.06 \pm 0.02 \mathrm{eV}, N_{\mathrm{t}}^{4}=4 \times 10^{15} \mathrm{~cm}^{-3}$ were found. It should be emphasized that the value of the trapping state density $N_{\mathrm{t}}$ does not include all local states, i.e. number of displaced molecules around the structural defect, e.g. edge dislocation. Such kind of structural defect includes about hundred and more molecules, but experimentally only the deepest trapping state of local state assembly emerges [3]. As we can see, the TM-SCLC method provides a relatively high resolution of the energy spectra of local trapping states of the order of \pm 0.02 to $\pm 0.05 \mathrm{eV}$. The obtained values of trap energy depth $E_{\mathrm{t}}=0.21$, 0.28 and $0.35 \mathrm{eV}$ are in good agreement with data obtained by the method of 


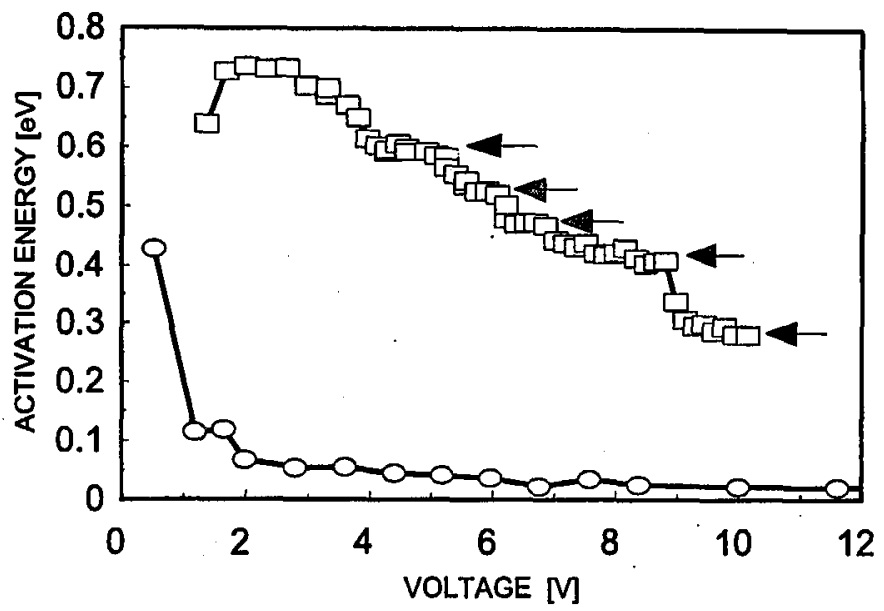

Fig. 4. Experimental $E_{\mathrm{a}}(U)$ dependences of the Au/IPB/Al (o) and Au/DMABI/Al (口) vacuum evaporated thin films. The temperature range $T=300-280 \mathrm{~K}$; the thickness of the IPB film $L=0.3 \mu \mathrm{m}$, of the DMABI film $-L=0.32 \mu \mathrm{m}$; injection of holes from positive $A u$ electrode. The arrows show the energy depth $E_{\mathrm{t}}$ of five sets of trap distribution for the DMABI films.

thermally activated currents [11]. On the other hand, the conventional method of SCLC gives instead of the set of three distributions a wide distribution curve with the maximum at $E_{\mathrm{t}}=0.36 \pm 0.04 \mathrm{eV}$ and $\sigma=0.05 \pm 0.03 \mathrm{eV}$ which actually envelops all three separate sets of trapping states [11]. More detailed description concerning calculation of trapping state parameters by SCLC of the Gaussian distribution of traps are given in $[1,3,8,11]$. As has been shown earlier by thermally stimulated current measurements of thin Pc films, there are also deeper sets of traps situated at $E_{\mathrm{t}}=0.47 \mathrm{eV}$ and $E_{\mathrm{t}}=0.68 \mathrm{eV}$ [17]. Most probably due to contact-limited processes these traps were not possible to observe by the TM-SCLC method.

In thin films of DMABI five sets of hole traps were observed with energies $E_{\mathrm{t}}^{1}=0.59 \pm 0.02 \mathrm{eV}, N_{\mathrm{t}}^{1}=8 \times 10^{15} \mathrm{~cm}^{-3} ; E_{\mathrm{t}}^{2}=0.52 \pm 0.02 \mathrm{eV}, N_{\mathrm{t}}^{2}=1 \times$ $10^{16} \mathrm{~cm}^{-3} ; E_{\mathrm{t}}^{3}=0.47 \pm 0.02 \mathrm{eV}, N_{\mathrm{t}}^{3}=1 \times 10^{16} \mathrm{~cm}^{-3} ; E_{\mathrm{t}}^{4}=0.42 \pm 0.02 \mathrm{eV}$, $N_{\mathrm{t}}^{4}=1.5 \times 10^{16} \mathrm{~cm}^{-3}$ and $E_{\mathrm{t}}^{5}=0.28 \pm 0.02 \mathrm{eV}, N_{\mathrm{t}}^{4}=1.6 \times 10^{16} \mathrm{~cm}^{-3}$. In our experiments it was not possible to examine whole energy gap up to conducting level of holes $E_{\mathrm{h}}$ and, consequently, to observe traps lower than $0.28 \mathrm{eV}$.

On the contrary, in thin polycrystalline films of polar compound IPB no hole traps in energy gap were observed. As seen from Fig. 4, the value of activation energy decreases with applied voltage. At high electric fields $\left(\mathcal{E} \approx 7 \times 10^{5} \mathrm{~V} / \mathrm{cm}\right)$ the value of $E_{\mathrm{a}}$ is close to zero $E_{\mathrm{t}}=0.02 \pm 0.01 \mathrm{eV}$. At still higher electric fields the value of $E_{\mathrm{a}}$ reaches zero value. Since the value of $E_{\mathrm{a}}=0.02 \mathrm{eV}$ in this case is close to $k T$ it is difficult to obtain more correct value.

In polycrystalline films of Pc and DMABI at low values of applied voltage the value of $E_{\mathrm{a}}$ increased with voltage (see Fig. 3 and Fig. 4). Such dependencies may be connected with contact-limited effects at the interface between electrode and 
organic layers. Thus, this high value of $E_{\mathrm{a}}$ at low electric fields is not connected with trapping states in the volume.

\section{Activation energy dependencies in LB films}

The TM-SCLC measurements of LB films demonstrated different behaviour of the activation energy dependencies on applied voltage $E_{\mathrm{a}}(U)$. As may be seen from Fig. 5 for VOPhc LB layers, the dependence $E_{\mathrm{a}}(U)$ of bulk conductivity (across the layers in a "sandwich" type cell) are more complicated, of oscillatory character, with a number of maxima and minima $[12,13]$. Similar dependence of

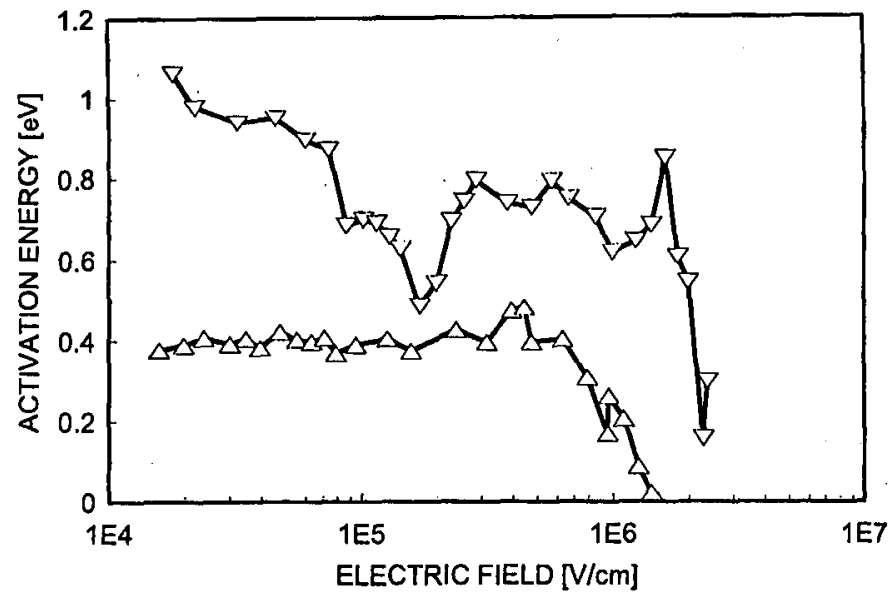

Fig. 5. Experimental $E_{\mathrm{a}}(\mathcal{E})$ dependencies of VOPhc $Y$-type LB multilayers in a "sandwich" type cell $\mathrm{Si}(p) / \mathrm{VOPh} / \mathrm{Bi}$ with different number $N$ of monolayers: $\Delta-N=30$, $\nabla-N=50$. The range of temperature $T=300-290 \mathrm{~K}$; the injection of holes from positive Bi electrode.

$E_{\mathrm{a}}(U)$ was observed also for C17IPB bulk conductivity (see Fig. 6) [18]. Such shape of $E_{\mathrm{a}}(U)$ curves does not follow the theory of TM-SCLC and does not allow to obtain in a simple way the values of energetic depth $E_{\mathrm{t}}$ of the set of traps. As shown in Fig. 5 the complexity of the dependence of $E_{\mathrm{a}}(U)$ is increasing with increasing number of monolayers in the LB film. It should be mentioned that increasing the number of monolayers the influence of contact-limited effects decreases and thus the experimental values better characterize the bulk properties of sample.

In case of in-plane conductivity in a surface type of cell (see Fig. 6) the $E_{\mathrm{a}}(U)$ dependence is more step-like and similar to typical dependencies in accordance with the TM-SCLC method theory (cf. Fig. 2). It is most probable that the complicated $E_{\mathrm{a}}(U)$ dependencies as in Fig. 5 and Fig. 6 indicate that the charge carrier traps possess a spatially non-uniform distribution. In case of bulk conductivity charge carriers may be tunnelling from one layer of molecular "heads" to another one through the aliphatic tails [13]. In other words, the electronic states which take part in charge carrier tránsport are determined by the layer of the 


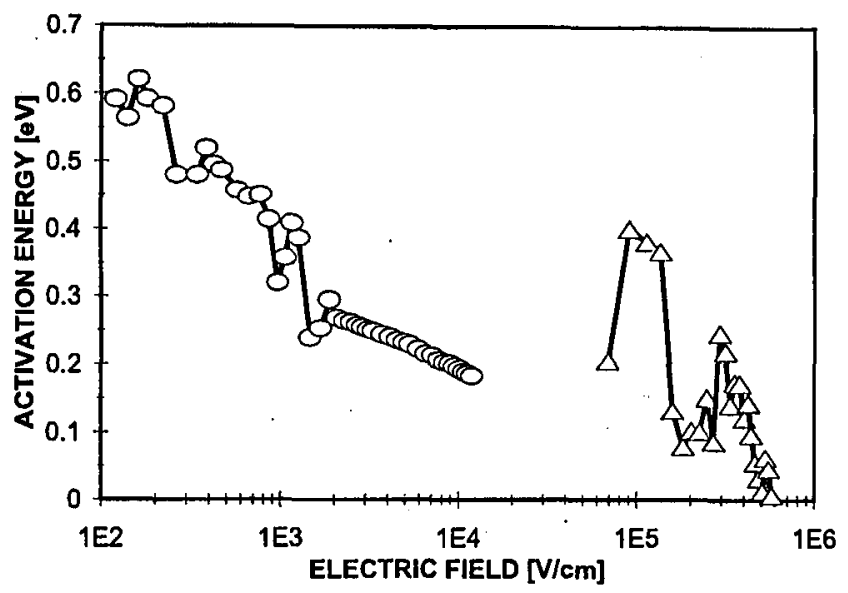

Fig. 6. Experimental $E_{\mathrm{a}}(\mathcal{E})$ dependencies of IPB $Z$-type LB multilayers system for bulk conductivity across the layers in a "sandwich" type cell $\mathrm{Cr} / \mathrm{C} 17 \mathrm{IPB} / \mathrm{Al}(\triangle)$ (the number of monolayers $N=24$ ) and for the in-plane conductivity of a surface cell Au/C17IPB/Au (o) with the distance between electrodes $150 \mu \mathrm{m}$. The injection of holes from $\mathrm{Cr}$ or $\mathrm{Au}$ electrodes.

polyconjugated molecular "heads" and are separated by layers of the aliphatic tails and, consequently, the trap distribution is actually determined by the quantum well structure of the LB films.

The observed trapping states in this case may be of structural origin. The structural investigation of VOPhc LB layers has shown that in LB films inside separate layers an amorphous structure dominates with inclusion of dispersive crystalline regions [19]. The crystalline domains with more closely packed molecules form, according to the polarization model $[1,3]$, charge carrier trapping states of structural origin. Thus, a spatially non-uniform distribution of trapping states may emerge.

It is interesting to notice that at sufficiently high electric fields $(\mathcal{E}>$ $1 \times 10^{6} \mathrm{~V} / \mathrm{cm}$ for VOPhc and $\mathcal{E}>5 \times 10^{5} \mathrm{~V} / \mathrm{cm}$ for C17IPB films) the activation energy $E_{\mathrm{a}}$ of bulk conductivity drops down to zero value $\left(E_{\mathrm{a}} \rightarrow 0\right)$ (see Fig. 5 and Fig. 6). This means that at these electric field values the bulk conductivity across the multilayer switches over to "trap-free" regime. It may be anticipated that at these field values hot, non-thermalized charge carriers are created [13].

If applied electric field is parallel to monolayers (the in-plane conductivity) the charge carrier may travel inside one monolayer of polyconjugated molecular "heads". In this case one may consider that the spatial distribution of traps is more uniform through the layer.

In LB layers of C17IPB several sets of hole traps were observed at $E_{\mathrm{t}}=$ $0.45 \pm 0.03$ and $0.18 \pm 0.03 \mathrm{eV}$ for in-plane conductivity and $E_{\mathrm{t}}=0.18 \pm 0.05$ and $E_{\mathrm{t}} \approx 0$ for bulk conductivity (see Fig. 6 ). These results are different from vacuum evaporated layers and may be caused by influence of the specific structure of LB films. On the other hand, in-plane activation energies of $L B$ films were measured at 
lower applied electric fields. In thin films of IPB such low values of electric field is not possible to apply to the sample since contact-limited effects emerge. Thus, the deeper sets of traps in energy gap were not detected in case of bulk conductivity.

\section{Conclusions}

We have demonstrated that the TM-SCLC method provides higher energy spectra resolution (up to $\pm 0.02 \mathrm{eV}$ ) in comparison with the conventional SCLC method. The TM-SCLC method is especially suitable for the studies of shallow structural trapping states of polarization origin possessing the Gaussian character of distribution. The main advantage of the method is that the energy spectra of several sets of trapping states can be directly obtained from the step-like structure of the $E_{\mathrm{a}}(U)$ dependence.

We have demonstrated that the TM-SCLC method works well in case of vacuum evaporated layers of different classes of OMC (Pc, IPB and DMABI polycrystalline layers). It is interesting to mention that in $\mathrm{Pc}$ and DMABI evaporated films a set of four or five shallow trapping state distribution emerge. In contrary to this, in case of IPB evaporated layers the energy gap is practically free from shallow traps. Only a single set of very shallow trapping states close to conductivity level can be detected. Such a behaviour of the IPB molecules in polycrystalline evaporated layers is rather unique and may be regarded as an additional peculiarity of this exotic high polar compound (cf. [16]).

The situation is more complicated in the case of LB multilayers. The $E_{\mathrm{a}}(U)$ dependencies in VOPhc and C17IPB films do not exhibit a typical step-like dependence but rather complicated curve of oscillatory character. This means that in this case the TM-SCLC theory is not applicable for the determination of trapping state energy spectra. As already mentioned this effect may be caused by non-uniform spatial trap distribution due to the quantum-well energy structure of LB multilayers. This phenomenon requires additional experimental and theoretical studies.

On the other hand, the in-plane conductivity of C17IPB LB films, measured in a surface cell, exhibits a step-like $E_{\mathrm{a}}(U)$ dependence and the corresponding trap distributions can be determined by the TM-SCLC method.

\section{Acknowledgment}

The authors acknowledge the financial support by the Soros International Science Foundation which allowed to perform the present studies.

We are also grateful to Dr. L. Gerca and Mrs. G. Matisova for the preparation of C17IPB LB films and Dr E. Fonavs for electrical measurements of DMABI thin films and C17IPB LB multilayers.

\section{References}

[1] E.A. Silinsh, Organic Molecular Crystals, Their Electronic States, Springer Verlag, Heidelberg 1980.

[2] M. Pope, C. Swenberg, Electronic Processes in Organic Crystals, Clarendon Press, Oxford University Press, Oxford 1982. 
[3] E.A. Silinsh, V. Čapek, Organic Molecular Crystals. Interaction, Localization and Transport Phenomena, AIP Press, New York 1994.

[4] F. Schauer, S. Nešpurek, O. Zmeškal, J. Phys. C, Solid State Phys. 19, 7231 (1986).

[5] S. Nešpurek, O. Zmeškal, F. Schauer, Phys. Status Solidi A 85, 619 (1984).

[6] F. Schauer, O. Zmeškal, J. Non-Cryst. Solids 164-166, 537 (1993).

[7] F. Schauer, O. Zmeškal, S. Nešpurek, Phys. Status Solidi A 75, 591 (1983).

[8] L.F. Taure, I.J. Muzikante, E.A. Silinsh, in: Potsdamer Forschungen der Pädagodischen Hochschule "Karl Liebknecht", Ed. H. Hänsel, Vol. 51, Pädagogische Hochschule "Karl Liebknecht", Potsdam 1987, p. 136.

[9] E.A. Silinsh, A.I. Belkind, D.R. Balode, A.J. Biseniece, V.V. Grechov, L.F. Taure, M.V. Kurik, J.I. Vertzymacha, I. Bok, Phys. Status Solidi A 25, 339 (1974).

[10] E.A. Silinsh, V.A. Kolesnikov, I.J. Muzikante, D.R. Balode, Phys. Status Solidi B 113,379 (1982).

[11] I.J. Muzikante, A.J. Rampans, L.F. Taure, Izv. Akad. Nauk Latv. SSR, Ser. Fiz. Tekh. Nauk 1, 76 (1986) (in Russian).

[12] I.H. Abele, D.Z. Ducmanis, I.J. Muzikante, T.F. Taure, E.A. Silinsh, Izv. Akad. Nauk Latv. SSR, Ser. Fiz. Tekh. Nauk 4, 15 (1989) (in Russian).

[13] E.A. Silinsh, I.J. Muzikante, L.F. Taure, G.A. Shlihta, J. Mol. Electron. 7, 127 (1991).

[14] L. Valkunas, D. Juodzballis, A. Urbas, A. Groudis, A.D. Durandin, E.A. Silinsh, A. Klimkans, S. Larson, Adv. Mater. Opt. Electron. 2, 221 (1993).

[15] N.A. Dimond, T.K. Mukherjee, Discuss. Faraday Soc. 51, 102 (1969).

[16] M.A. Rutkis, L.E. Gerca, E.A. Silinsh, O.Y. Neilands, M.P. Roze, E.L. Berzinsh, A.B. Klimkans, S. Larson, Adv. Mater. Opt. Electron. 2, 319 (1994).

[17] I.J. Muzikante, A.J. Rampans, E.A. Silinsh, L.F. Taure, Izv. Akad. Nauk Latv. SSR, Ser. Fiz. Tekh. Nauk 4, 18 (1983) (in Russian).

[18] I. Muzikante, E.A. Silinsh, in: 5th European Conf. on Organic Films, Smolenice Castle (Slovak Republik) 1994, Ed. R.H. Tredgold, Slovak Academy of Sciences, Bratislava 1994, p. 63.

[19] V.I. Troitsky, V.I. Bannikov, T.S. Berzina, J. Mol. Electron. 5, 147 (1989). 